\title{
ARTE Y AFECTIVIDAD EN LA EXPERIEN- CIA ESCOLAR INCLUSIVA: UN ESTUDIO DOMINICANO
}

Art and affectivity in an inclusive school experience: A Dominican Study

\section{Aurora Andreína Jiménez Soto Rocío Hernández Mella Patricia Liranzo Soto Berenice Pacheco-Salazar1}

Resumen: Arte y afectividad en la experiencia escolar inclusiva: un estudio dominicano corresponde al primero de una serie de cinco artículos surgidos de una investigación con un alcance exploratorio, enfocada en la descripción de la experiencia educativa de niños y niñas en interacción con sus docentes. Se trabajó con 123 estudiantes del nivel primario y diecinueve docentes, de cuatro centros educativos públicos en el municipio de Yamasá, República Dominicana. En esta oportunidad, se presentan los objetivos del estudio, el marco teórico que lo sustenta y el método de investigación llevado a cabo.

Palabras clave: arte, afectividad, escolaridad primaria.

Esta investigación contó con la participación activa de estudiantes de término de la Carrera de Psicología, adscrita al Área de Ciencias Sociales y Humanidades, del Instituto Tecnológico de Santo Domingo (INTEC): Mariano Soto, Maffel Santana, Triny Mejía, Mirelys de los Santos, Susary Vargas y Elía Rijo. 
Aurora Andreina Jiménez. Soto, Rocío Hernández. Mella, Patricia Liranzo Soto, Berenice Pacheco-Salazar

\begin{abstract}
Art and Affectivity in an Inclusive school experience: A Dominican Study corresponds to the first article of a series of five emerged from research done with an exploratory reach, and focusing on the description of the educative experience with boys and girls in interaction with their teachers. The study was done with 123 elementary students and 19 teachers from four public schools in the municipality of Yamasá in Dominican Republic. In this opportunity, the objective of the study, the theoretical framework and the research method are presented.
\end{abstract}

Keywords: Art, affectivity, attitude, elementary school.

\title{
1. Introducción
}

El presente artículo se ha elaborado a partir de una investigación inscrita en las corrientes de la psicología socioeducativa, con el fin de contribuir a mostrar los beneficios del vínculo entre el arte y la afectividad en el proceso enseñanza-aprendizaje. Por esto, se defiende que la experiencia educativa parta de las propias capacidades creativas, de los recursos y el estado personal tanto del estudiantado como de las docentes, potenciando así su valor dentro de la escuela.

Este primer artículo presenta una síntesis del marco teórico conceptual que sirvió de punto de partida del estudio, así como la metodología de investigación llevada a cabo. Los artículos subsiguientes irán presentando los principales hallazgos encontrados y los retos que estos representan para el sistema educativo dominicano. 


\section{Objetivos del estudio}

\subsection{Objetivo general}

Describir la experiencia educativa de niños y niñas de 1ero. a 4to. grados del Nivel Básico de escolaridad, en interacción con sus docentes, para el establecimiento de una estrategia lúdica y creativa que aporte al desarrollo de las potencialidades de todos los actores involucrados.

\subsection{Objetivos específicos}

- Identificar las disposiciones personales y diversas de los niños y niñas en su experiencia escolar.

- Determinar la expresión de sentimientos de los niños y niñas en su relación con los contextos personal y escolar en que se desenvuelven.

- Describir las actitudes de los niños y las niñas frente a la lectura.

- Describir las actitudes de los niños y las niñas frente a las clases de matemática.

- Identificar las actitudes, acciones y recursos educativos utilizados por los y las docentes en la experiencia escolar.

- Propiciar estrategias de intervención, lúdicas y artísticas, para complementar y apoyar el proceso enseñanza-aprendizaje de los niños y niñas.

\section{Marco teórico}

\section{1 $\mathrm{El}$ arte en la experiencia educativa}

Es imperativo hoy en día promover una formación integral en las escuelas, en vista de las demandas tan complejas que la sociedad hace a sus individuos y lo es más cuando se trata de poblaciones 
Aurora Andreina Jiménez. Soto, Rocío Hernández. Mella, Patricia Liranzo Soto, Berenice Pacheco-Salazar

provenientes de hogares desaventajados, para quienes la escuela viene a ser la única oferta educativa con la que disponen. Esta formación integral incluye la educación artística y la educación a través del arte como ejes clave para lograr la equidad de oportunidades y el desarrollo pleno de las capacidades.

Más aún, el arte constituye el instrumento ideal para reinventar la función docente. "¿De qué forma es posible conjurar la crisis epocal por la que atraviesa la escuela? A través de la "educación artística y la educación por el arte”, asegura Lucina Jiménez (2010), quien llega a defender el rol que tiene la educación en contrarrestar no solo las formas tradicionales de analfabetismo sino también "nuevas formas de analfabetismo y de exclusión social... entre ellas, la del analfabetismo estético... (que) confina a los niños y jóvenes a una sola dimensión de la vida... y al papel de consumidores de un mundo de imágenes y de productos culturales" (pp. 206 y 213).

De acuerdo con lo postulado por Jiménez (2010) es incuestionable el poder del arte para transformar a las personas y generar valores. A lo dicho se suma el impacto a veces inmediato en la salud emocional, afectiva y física que tiene el arte en los niños, niñas, adolescentes y adultos nos lleva a propugnar por una educación a través del arte, como vía idónea del desarrollo de capacidades y la formación de ciudadanía.

Eduardo Villanueva (2012: 1) expone que “diversos estudios y experiencias en escuelas de Latinoamérica han demostrado que la danza, por su valor pedagógico y como estrategia de enseñanza de competencias sociales y actitudes positivas hacia la vida, mejora el proceso de aprendizaje escolar. La educación con la danza también contribuye a disminuir la violencia intrafamiliar y la violencia social al fomentar el respeto por sí mismo y por el otro".

Asimismo, la creatividad es central en los planteamientos que formula Marcos Villamán (2003) sobre la calidad en la educación, creatividad que la imaginación libera, utilizando la incertidumbre como oportunidad y a través de la educación artística. 
Arte y afectividad en la experiencia escolar inclusiva:

Un estudio dominicano

Una experiencia educativa organizada alrededor de actividades artísticas desarrolla las capacidades humanas, libera emociones y necesariamente se enriquece a partir de los aportes de cada uno y una de sus actores. Además, se aprende a construir una vida en común cuando aceptamos la riqueza de cada persona en nuestras vidas.

Con la autora María Teresa Pozzoli (2007), sostenemos que el arte también tiene un poder reconstructor, ya que al constituirse en una vía de expresión de nuestra interioridad resulta sanador. Lo es, por el hecho de poder reconocer nuestro ser profundo y poder expresarnos desde ese nivel de existencia con alguno de los recursos simbólicos que el arte provee.

El arte abre las vías de comunicación al interno de un colectivo humano, propiciando las expresiones verbales y no verbales de los individuos. Sin embargo, para muchos adultos y jóvenes e incluso niños, el arte no es parte de sus vidas, lo que lleva a Badilla (2011) a explicar que tal parece que a las personas se les olvida cómo practicar el arte. Conjuntamente con este autor, defendemos que el arte es de vital importancia para los seres humanos y que tiene incidencias positivas en la salud y el camino hacia la felicidad.

Siempre debe haber oportunidades para actividades recreativas y artísticas con las cuales trabajar de manera compartida con otras aulas, otros cursos e incluso otros centros educativos. Esto daría un carácter flexible a la experiencia de aula, promoviendo el aprendizaje de forma muy original, y dando paso a la creación de valores encaminados a un desarrollo social de relaciones democráticas. Expresado de forma sencilla, entendemos que una estrategia educativa desde el arte provocaría en el estudiantado deseos de estar presente en su escuela. De esta manera, dejaríamos de escuchar frases como: ya nos soltaron, a la hora que van al recreo o cuando terminan sus labores educativas.

De acuerdo con Abad (2009), la educación a través del arte representa en la actualidad un puente que integra y conecta las diferentes 
Aurora Andreina Jiménez. Soto, Rocío Hernández Mella, Patricia Liranzo Soto, Berenice Pacheco-Salazar

realidades que se comparten, y no únicamente una manifestación particular del espíritu humano. El arte como ejercicio educativo horizontaliza la cultura haciendo posible que todos y cada uno de nosotros pueda construirla. Esta función socializadora del arte, más allá de lo estético, es un pilar para el cambio de las sociedades de una manera progresiva. La educación a través del arte posibilita un espacio de búsqueda, de reflexión y responsabilidad, tanto de forma colectiva como individual. Esta intervención se convierte en una plataforma que consolida el arte y la afectividad en su función socializadora e integradora de saberes.

\subsection{La escuela y el aula como espacios afectivos}

$\mathrm{El}$ arte hace visible procesos que se viven internamente, trayendo a la luz emociones que, de otro modo, permanecerían ocultas. De ahí que Grajales (2011) se exprese defendiendo el arte como un medio ideal para expresar aquellas fuerzas o conflictos internos que con palabras no resulta fácil pronunciar. De este modo se hace evidente un vínculo estrecho entre el arte y la afectividad.

Al hablar de lo afectivo se hace alusión a lo postulado por Bach (2002: 2) quien afirma que la dimensión afectivo-emocional es el fundamento del desarrollo holístico, explicándolo como: "síntesis entre fisiología-sensación, cognición-pensamiento y comportamiento-acción, punto de unión entre lo interno o biológico y lo externo o social”. (Citado por Hernández, 2012).

El estado emocional tiene su asiento en el cuerpo y es este cuerpo la puerta de entrada al proceso de reflexión del mundo emocional de una persona. Educar lo emocional, entonces, significa el reencuentro con uno mismo en condición de total aceptación, como lo expresa Maturana (1990; 1992): "La emoción que constituye la coexistencia social es el amor, esto es, el domino de aquellas acciones que constituyen a otro como legítimo otro en coexistencia con uno..." (Citado por Hernández, 2012: 2). 
La afectividad constituye una fuerza de vida. Piaget $(1975,1986)$ reconoce que no existe ningún acto humano desprovisto de energía afectiva y que hay un paralelismo entre el desarrollo cognoscitivo (o de la conciencia) y el afectivo. En su acepción del término interés, el autor comprende tanto una energía afectiva como una acción cognoscitiva y en cada etapa del desarrollo habría que conocer cómo se expresan antes de etiquetar un comportamiento como negativo o disminuido. Dentro del espacio psíquico en el cual conviven los seres humanos, el desarrollo afectivo-moral resulta central a la hora de conceptualizar los aspectos esenciales que forman parte integral de la persona.

Considerar la afectividad como elemento sustantivo del proceso de adquisición de conocimiento es también explicado por autores como Delors (1996), Faure (1997) y Morin (2000) (citados por Restrepo, 2009), quienes plantearon que la concepción de una educación más amplia y flexible no podría estar completa sin la dimensión afectiva.

De esta manera, la experiencia educativa debería destacar el sentido de totalidad del ser humano, es decir, el desarrollo de cada individuo incluyendo lo somático, lo cognitivo, lo afectivo, lo social, y lo espiritual, y sus potencialidades en cada aspecto. El reto actual del contexto educativo sería "recuperar el alma sensible, la presencia, la valoración y expresión de las emociones, los sentimientos y la ternura en una cultura de auténtica convivencia y respeto". (Villegas, 2010: 6).

La escuela es un entorno donde la afectividad se expresa a través de las relaciones interpersonales, pero el desarrollo de estas últimas necesitará ser establecido, mantenido y alimentado. Dichas relaciones brindan soporte emocional, físico y de información, sin embargo, como lo señala De Zubiria (2007, citado por Villegas, 2010), cada vez es más difícil que los niños y adolescentes sean capaces de comenzar, ahondar y resolver conflictos en sus relaciones personales. En este tenor, Rodríguez (2010) explica que para lidiar 
Aurora Andreina Jiménez Soto, Rocio Hernández. Mella, Patricia Liranzo Soto, Berenice Pacheco-Salazar

con las emociones adecuadamente es necesario reconocerlas y nombrarlas, para luego poder reflexionar y conversar sobre ellas, así como compartirlas con las personas que nos rodean.

Rodríguez (2010) amplía que, a medida que los seres humanos se desarrollan, el control sobre las emociones se lleva a cabo de forma interna más que externa, aunque el grupo siempre representará un peso dentro de las decisiones individuales. Sin embargo, en el caso de lo encontrado por De Zubiría (2007, citado por Villegas, 2010), este autor explica que la dificultad que se viene observando tiene que ver con el nivel de sofisticación y complejidad de las propias relaciones personales, ya que en la enseñanza estas implican el contacto consigo mismo y con el otro. Este último autor expresa que la manera de desarrollar las relaciones, sobre todo las que tienen que ver con los demás, se dan en virtud de tres acciones: conocer, valorar e interactuar.

Una de las estrategias para que este accionar planteado por De Zubiria (2007) sea posible, lo muestran las técnicas lúdicas y artísticas, o sea, la educación a través del arte que ofrece vías de expresión a esa afectividad única y particular de cada persona y propicia una visión y aceptación de toda la diversidad que caracteriza al ser humano.

La dimensión afectiva debe hacerse relevante en el espacio educativo y organizacional de la escuela. En el aula significa rescatar y cimentar el aprendizaje de la afectividad sobre la experiencia cotidiana de las personas, en sus intercambios particulares y sociales, mientras se produce el proceso aprendizaje-enseñanza cognoscitivo. Puede decirse que, más que un área, la afectividad sería una estructura continua del accionar educativo. 


\subsection{Actitud: factor relevante en el cambio educativo}

En consonancia con lo dicho anteriormente, nos encontramos con el reto de que la escuela trabaje desde el reconocimiento de las actitudes de su estudiantado.

La actitud es uno de los aspectos de la personalidad de un individuo y un elemento influyente en la manera de comportarse de los seres humanos.

Una actitud es una organización relativamente estable de creencias, sentimientos y tendencias hacia algo o alguien. Las creencias incluyen hechos, opiniones y nuestro conocimiento general acerca del objeto. Los sentimientos abarcan odio, amor, agrado, desagrado y otros sentimientos similares. Las tendencias de conductas se refieren a nuestras inclinaciones para actuar de cierta manera hacia ese objeto. (Morris \& Maisto, 2005: 421).

Las actitudes son desarrolladas en el proceso de crecimiento de un ser humano, se adquieren en la interacción social que tiene lugar en los diferentes espacios de socialización del individuo, como son la familia, la escuela y la comunidad. Dicho de otro modo, por medio al aprendizaje se hace posible algún tipo de cambio o modificación en las actitudes, sin perder de vista que muchas de estas disposiciones actitudinales son bastante estables en el tiempo.

El concepto de actitud presenta muchas definiciones. Solo Allport (citado por Ortego, López \& Álvarez, 2011) agrupó 100 definiciones distintas, lo que expresa la ambigüedad del mismo, sin embargo, al revisar estas aproximaciones de lo que es una actitud se encuentran varios puntos comunes en todos los conceptos, que son:

- Actitud como una predisposición o conjunto de predisposiciones

- Actitud como una predisposición aprendida 
Aurora Andreina Jiménez, Soto, Rocío Hernández. Mella, Patricia Liranzo Soto, Berenice Pacheco-Salazar

- Actitud como una predisposición con cualidades opuestas, en positivo o negativo, etc.

- Actitud como predisposición hacia algo o alguien.

- Actitud como una predisposición que forma parte de la estructura de un estado mental.

La actitud está compuesta por tres aspectos generales: el afectivo, el cognitivo y el conductual. El componente afectivo se refiere a las emociones y sentimientos que experimenta y demuestra una persona frente a aquello que la estimula, ya fuese otra persona, circunstancia, tiempo, lugar, etc. El componente cognitivo es el juicio, criterio, o idea que tiene una persona frente a un estímulo o conjunto de estímulos. El componente conductual se refiere a la manera de actuar o comportarse que tiene la persona a partir de presentársele o interactuar con el estímulo. Estos tres componentes funcionan de manera interrelacionada. Es importante destacar que un aspecto fundamental en las actitudes es el componente evaluativo-afectivo, debido al encuentro que se da entre las normas establecidas por el medio ambiente y las normas subjetivas que desarrolla cada persona, las cuales están cimentadas en la afectividad (Prieto, 2011).

Las actitudes tienen diferentes funciones en la vida psicológica de una persona, ellas apuntan a actuar como autodefensas, colaboran con la función adaptativa del individuo, como el logro de objetivos $y$, además, las actitudes funcionan como expresiones de conocimiento y de valores (Sánchez \& Mesa, 1998).

Una de las funciones de una escuela inclusiva e integral, es "trabajar la participación, la responsabilidad, el respeto a los derechos de los demás, la tolerancia y el sentido crítico, que configuran las actitudes que resultan básicas para la convivencia democrática" (Sánchez \& Mesa, 1998: 31). Así pues, esta concepción, de considerar las actitudes personales y sociales como un factor relevante en la convivencia humana, debe ser incluida en la planificación y desarrollo de la experiencia educativa, sobre todo en la práctica de aula. 
Actualmente, en el espacio social existe una preocupación generalizada sobre qué significa educar en valores y fomentar ciudadanos y ciudadanas con actitudes positivas hacia la vida en general, a pesar de las circunstancias particulares, y de poder trabajar en el cambio de aquellas actitudes negativas que se manifiestan en los múltiples entornos donde interactúan los individuos (Sánchez \& Mesa, 1998).

Establecer propuestas que apunten a trabajar con las actitudes es un reto desde los planteos de la afectividad, como eje de desarrollo y parte esencial en la construcción de conocimiento, que nos atrevemos a sugerir que la educación a través del arte nos permite hacer de la acción educativa una experiencia inclusiva y diversa, ya que legitima el sentido emocional de nuestra cotidianidad y convivencia, lo que nos hace humanos.

\subsection{Diversidad e inclusión: claves en la calidad educativa}

Hasta el día de hoy, la escuela como institución organiza la experiencia educativa de manera homogénea y utiliza la edad para dividir a sus estudiantes. ¿Por qué ocurre esto? ¿Cómo se da el desarrollo y qué papel juega la edad en él? Además, ¿'se justifica que, por ser de la misma edad, los niños y las niñas de un curso sean tratados como iguales? Hay algo que resulta muy obvio y, por lo mismo, pasa desapercibido: los muchos aspectos y cualidades físicas que poseemos. ¿No sería esta diversidad física responsable de comportamientos también distintos? La experiencia educativa implica la totalidad de la persona, y la persona es mucho más que su cuerpo y su conducta. Nos encontramos, entonces, ante una situación muy compleja.

Para explicar el desarrollo, el biólogo chileno Humberto Maturana (1992) nos invita a reconocer el acoplamiento entre la estructura corporal y el espacio psíquico y cómo ambos se modulan entre sí en el vivir humano. Por lo tanto, no existe psiquis sin cuerpo ni manera 
Aurora Andreina Jiménez. Soto, Rocío Hernández. Mella, Patricia Liranzo Soto, Berenice Pacheco-Salazar

de conocer el cuerpo sin la psiquis, a la hora de explicar la vida de los seres humanos.

Jean Piaget (1975,1986), biólogo y epistemólogo suizo, explicaba que son cuatro tipos de factores a través de los cuales se produce el desarrollo mental o de la conciencia de la persona: maduración, experiencia con los objetos, transmisión social y equilibración. El primero fue abordado más arriba, el segundo se refiere al involucramiento directo con el mundo, el tercero alude a la vida social, de la cual la escuela es parte. El cuarto factor expone un mecanismo autorregulador que ocurre en la mente y que da como resultado un conocimiento organizado que varía cualitativamente en el tiempo. A estas variaciones Piaget $(1975,1986)$ las llamó etapas y cada una abarca varios años y aptitudes para conocer.

Explicando también la mente tenemos al estadounidense Howard Gardner (1983,1993), psicólogo, quien ha desarrollado una línea de investigación sistemática para mostrar que la mente no es una capacidad general, sino capacidades específicas que, aunque relacionadas, son independientes unas de otras. Se trata de la teoría de las inteligencias múltiples, que viene a hacer más complejo el panorama que dejó Piaget. Cada persona tiene una o más de una inteligencias predominantes y las otras de menor relieve. Gardner ha explicado, hasta el momento, ocho inteligencias, pero afirma que habría muchas más. Las inteligencias son: Cinético-corporal o Kinestésica, Lingüística, Espacial, Intrapersonal, Interpersonal, Lógicomatemática, Musical y Naturalista.

Además de las inteligencias, los niños y las niñas tienen diferentes estilos y ritmos de aprendizaje, que están asociados a su propia personalidad. También pueden existir diferencias que tengan que ver con los entornos sociales y culturales donde se han criado los niños y las niñas, que deben ser tomados en cuenta en la experiencia educativa para desarrollar así la inteligencia, la creatividad y autoestima. 
El asunto sería entender la educación como promotora de la diversidad, aceptando que cada persona tiene talentos diferentes para comprender su mundo y a sí misma, lo mismo que para expresarse; más que quedarse en una preocupación generalista del desarrollo de los procesos superiores o resumirlos a los consabidos de capacidad lingüística y capacidad lógica, tal y como critica Howard Gardner (1997) al postular su teoría de las inteligencias múltiples.

Al maestro y a la maestra les corresponde promover espacios y las condiciones para evitar la segregación entre el estudiantado. Para ello, se hace fundamental la asunción y práctica de principios como la solidaridad, respeto, libertad, participación y dignidad humana.

La educación inclusiva es, ante todo, una cuestión de justicia y de igualdad, ya que aspira a proporcionar una educación de calidad para todas las personas sin distinción de condición física, mental ni social. Se concibe la educación inclusiva como un derecho humano con un sentido tanto educativo como social, al tiempo que rechaza que a los sistemas educativos tengan derecho solo cierto tipo de niños.

Para Tony Booth (s/f) y Mel Ainscow (2003), especialistas en educación y escuelas inclusivas, la inclusión es un conjunto de procesos orientados a aumentar la participación de toda persona estudiante en la cultura, los currículos y las comunidades de las escuelas.

Para estos autores, la inclusión implica que los centros realicen un análisis crítico sobre lo que pueden hacer para mejorar el aprendizaje y la participación de todos y todas. Esto incluye el derecho de toda persona a ser escuchada y a tener en cuenta sus opiniones sobre los diferentes aspectos que afectan su vida y que involucra los procesos democráticos de toma de decisiones, en los que están implicados diferentes actores de la comunidad educativa, incluyendo el estudiantado. 
Aurora Andreina Jiménez. Soto, Rocío Hernández Mella, Patricia Liranzo Soto, Berenice Pacheco-Salazar

Atender la diversidad produce una educación inclusiva, que consiste en un proceso de aumentar la participación de toda persona en la escuela. Esto se da a partir de reconocer el derecho a la educación, que toda persona puede y debe aprender, que la diversidad es una característica de lo humano. Para lograrlo, se requiere de un cambio paradigmático en el modo en que se observan las llamadas dificultades educativas, logrando que toda persona estudiante que actualmente se cataloga como estudiante con necesidades especiales pase a ser considerada como el estímulo que puede fomentar desarrollos hacia un entorno de aprendizaje más rico (Ainscow, 2003).

\subsection{Aprendizaje y su vinculación con la atención a la diversidad}

Las aulas de clase son un espacio donde se dan continua y permanentemente manifestaciones de la diversidad de los alumnos y las alumnas que las conforman. Y una manifestación patente de estas diferencias que caracterizan el comportamiento humano la encontramos en la multiplicidad de respuestas ante una misma situación de aprendizaje. El aprendizaje no ocurre al margen de las capacidades cognoscitivas de cada ser humano y, cuando se trata de promover significado en los estudiantes, es imperativo partir de estas disposiciones en la experiencia escolar, si bien reconociendo el efecto que ejerce la presión social, tanto la de compañeros de grupo, como la que efectúa la intención pedagógica del docente (Hernández Mella, 2014).

Aunque estas diferencias siempre han existido, no siempre han sido tenidas en cuenta por el sistema educativo ni por los diferentes actores que participan en él. La escuela ha llevado a la práctica un tratamiento educativo homogeneizante en nombre de una supuesta efectividad y/o rentabilidad de recursos, y la labor del personal de psicología y orientación se ha centrado en garantizarla y detectar a quienes se desvían de ella. 
La diversidad resulta ser una característica de la condición humana que se manifiesta en el comportamiento y modo de vida de los individuos, así como en sus maneras de pensar; circunstancia esta que se da en todos los niveles evolutivos de la vida y en todas las situaciones, determinando las posibilidades de la experiencia educativa.

En este tenor, cabe preguntarse ¿qué significa sociedad bumana diversa?, ¿cuáles implicaciones tiene la asunción de la diversidad? Significa el reconocimiento e implica la aceptación de cada ser humano como un ser rico en capacidades, en una personalidad única y en una visión diferente acerca de lo que es vivir y aprender. Entre las personas existen diferencias, no solo en su apariencia física, sino en su forma de pensar, sentir, hacer y relacionarse con el mundo. Todo esto debe ser considerado, analizado y aprovechado para la experiencia educativa. En una escuela inclusiva se entiende que son estudiantes todos los niños y todas las niñas, incluyendo quienes tienen necesidades educativas especiales. No se considera a nadie como impedido o discapacitado o en desventaja, sino diferente (Hernández \& Pacheco, 2012).

La experiencia educativa que se deriva de esta concepción de diversidad, primero, no se enfoca en enseñar sino en promover el desarrollo de las capacidades y, a partir de ellas, orientar el aprendizaje a través de acciones significativas para quienes aprenden, y, segundo, es necesariamente flexible, pues se trata de atender la diversidad que nos conforma como personas, como los seres humanos que somos.

La experiencia recién reseñada implica dejar atrás la imagen de las clases centradas en lo que el maestro o la maestra tiene que decir. Es necesario construir un aula dotada de sentido, donde exista la posibilidad de compartir, debatir y disfrutar, en contextos donde se valoran, toman en cuenta y respetan sus diferencias individuales, y en la cual existe de manera permanente la oportunidad de aprender a través de los trabajos cooperativos. 
Aurora Andreina Jiménez. Soto, Rocío Hernández. Mella, Patricia Liranzo Soto, Berenice Pacheco-Salazar

Esto, por supuesto, plantea grandes retos a los sistemas educativos y nos lleva a buscar nuevas estrategias de formación del personal, tanto docente como de orientación y psicología, y de desarrollo del proceso enseñanza-aprendizaje. Así, el aula se convierte en un espacio de crecimiento para todas las personas involucradas, y se permite a las y los estudiantes apropiarse de una visión más cercana a sus propias existencias sociales y culturales.

Lo primero es que se comprenda que estar en la escuela es para hacer actuar la imaginación y fomentar valores, que las temáticas que se trabajan en el aula son solo ocasiones para que la imaginación se ejercite. En una escuela inclusiva, la planificación docente va más allá de las temáticas pedagógicas. Es muy importante que se planifiquen y desarrollen actividades artísticas y recreativas que estén integradas a temas curriculares o actividades libres propuestas por los propios estudiantes, en lo que se refiere al fomento del aprendizaje. Es decir que, a través de distintas actividades recreativas y artísticas, se comprende que en la escuela se va a disfrutar.

\subsection{Precisión del estado del arte}

Concebir la educación a través del arte como un eje transversal del proceso aprendizaje-enseñanza implica un posicionamiento distinto frente a la experiencia educativa; sin embargo, esta visión de construir el conocimiento presenta pocas experiencias que estén sistematizadas y publicadas. En este sentido, se expondrán algunas investigaciones que han utilizado la modalidad del arte para la enseñanza en general.

Uno de los hallazgos de la investigación realizada por Eddie Santiago (2009) titulada Música como estrategia educativa en el proceso enseñanza aprendizaje para el aprovechamiento de los estudiantes del nivel elemental en la región educativa de Bayamón, en Puerto Rico, expuso que la música es una intervención educativa que genera un mejor 
Arte y afectividad en la experiencia escolar inclusiva:

Un estudio dominicano

aprovechamiento académico debido a que posibilita que las habilidades cognoscitivas, psicomotoras y afectivas se desarrollen más ampliamente.

Otra investigación realizada por Verenice Tirado (2008) llamada El proceso de enseñanza aprendizaje a través de las artes plásticas en la educación pre-escolar indica que por medio a las artes plásticas el niño y la niña aprenden en la acción, es decir, cuando ejecutan actividades como observar, crear o manipular objetos. Estas ejecuciones, a su vez, posibilitan los aprendizajes significativos, no solo en los propósitos del programa, sino en todos los ámbitos del currículo escolar, como son: el lenguaje, la matemática, lo social y lo natural, sobre todo cuando se aspira a tener una educación integral.

En la investigación The impact of storybooks on kindergarten children's mathematical achievement and approaches to learning, de Jane Keat y Jane Wilburne (2009), los resultados expresan que los infantes, mientras estaban imaginando cómo resolver los conflictos de los personajes en los diferentes escenarios de los cuentos, eran capaces de utilizar el pensamiento matemático como una forma natural de ayudar a concluir la historia. Con cada discusión imaginada, los niños estaban construyendo conocimiento matemático y perfeccionando sus habilidades matemáticas de una forma que pueda asegurar ambos, tanto el rendimiento y las actitudes positivas, como los enfoques de aprendizaje de las matemáticas.

Los resultados de esta investigación pueden motivar a los maestros de niños pequeños a reflexionar acerca de la importancia de incluir múltiples lecturas de cuentos a los contenidos asociados con la matemática. De esta manera, los personajes de las historias y los contextos proporcionan a los niños pequeños conocimientos que fomentan el entusiasmo, aseguran el compromiso y justifica el tiempo invertido en clases. Otra implicación educativa a considerar es el beneficio derivado de la integración de los libros de cuentos a la matemática, la alfabetización, y los estudios sociales. 
Aurora Andreina Jiménez, Soto, Rocío Hernández. Mella, Patricia Liranzo Soto, Berenice Pacheco-Salazar

\section{Método}

Esta investigación es un estudio no experimental de tipo longitudinal-panel, con un enfoque mixto específico y con un alcance de tipo exploratorio y descriptivo.

El diseño longitudinal de panel surge de la medición de la variable en varios momentos en el tiempo y con el mismo grupo de sujetos; en este caso, la medición se efectuó en dos puntos en el tiempo: al principio de la recolección de los datos y después de la intervención psicoeducativa, a un mismo grupo de niños, niñas y docentes que conformaron la muestra del estudio.

Dada la manera de aproximarnos al tema de investigación, se utilizó el Diseño Transformativo Concurrente DISTRAC (Hernández-Sampieri, et al., 2010) que implica la recolección de datos cuantitativos y cualitativos en un mismo momento, es decir, concurrentemente. Este diseño posibilita que se conceda igual peso o importancia tanto a lo cuantitativo como a lo cualitativo.

\subsection{Contexto del estudio}

En la República Dominica, las estadísticas correspondientes al sector educativo indican que, con respecto a la cobertura neta en el Nivel Básico o primario, para el año 2010-2011 fue de un 95\% [Ministerio de Economía, Planificación y Desarrollo (MEPyD), Consejo Nacional para la Niñez y Adolescencia (CONANI) y Fondo de las Naciones Unidas para la Infancia (UNICEF)]. Como es evidente, el acceso a la escuela responde a los objetivos trazados, sin embargo, persisten dificultades en el indicador de calidad educativa.

Nuestra propuesta de Arte y Afectividad se concentró en el municipio de Yamasá, propio del distrito educativo 17-01, de la Regional Educativa de Monte Plata, el cual cuenta con 72 centros educativos. Nuestra investigación trabajó con cuatro (4) de ellos. 
Arte y afectividad en la experiencia escolar inclusiva:

Un estudio dominicano

\subsection{Muestra}

El presente estudio trabajó con una muestra de 123 estudiantes del nivel primario, niños y niñas de 1ro. a 3er. grado (año escolar 20132014), y posteriormente con esos mismos estudiantes (estando en los grados de 2do. a 4to.) en el año escolar 2014-2015. Estos fueron seleccionados a través de un muestreo no probabilístico de tipo intencional.

Asimismo, se trabajó con 19 docentes responsables de los mismos grados y aulas a los cuales asistían los niños y niñas seleccionados, pertenecientes a cuatro (4) centros de educación pública primaria de la Regional educativa de Monte Plata, Distrito Educativo de Yamasá.

\subsection{Instrumentos cuantitativos}

Los instrumentos utilizados fueron:

- Instrumento de Evaluación Psicopedagógica (IEP) de Hernández, R., Andújar, C., Scheker, A., Pérez, M. y Amiama, C. (2000), cuyo propósito es conocer las capacidades, intereses y habilidades cognoscitivas y afectivas de niños y niñas en interacción con los diferentes contextos que intervienen en la experiencia educativa. Además, valorar la intervención de otros actores dentro de cada contexto, como son: maestros y maestras, la familia, y los compañeros y compañeras. El instrumento se divide en cuatro componentes: Contexto Escuela, Contexto Familia, Contexto Niño o Niña y Competencias Curriculares. En esta investigación se utilizaron los primeros tres componentes, porque es allí donde se valoran las disposiciones cognoscitivas y las expresiones afectivas de los estudiantes en interacción con los demás actores de la experiencia escolar. En el instrumento, los ítems son respondidos de dos formas: la primera es el llenado de una lista de cotejo y la segunda forma, de preguntas abiertas. 
Aurora Andreina Jiménez. Soto, Rocío Hernández. Mella, Patricia Liranzo Soto, Berenice Pacheco-Salazar

- Valoración del Desarrollo Socio-Afectivo (de Hernández, \& Andújar, 1999). Este cuestionario pretender evaluar la dimensión socioafectiva en dos grandes áreas, la expresión de sentimientos y la valorización de acciones. Para esta investigación solo se utilizó el aspecto relativo a la expresión de sentimientos. La niña o el niño frente a una historia presentada de manera visual o escrita debía reconocer la emoción implicada en la misma. Para los niños y niñas más pequeños se le presentaban como opciones de respuestas caritas con expresiones de diferentes emociones. Para el caso de los niños y niñas mayores se les hacía una pregunta abierta acerca de lo que pudiera sentir el personaje.

- Cuestionario de actitud hacia la lectura en el nivel básico (ERAS) por sus siglas en inglés: The Elementary Reading Attitude Survey (de McKenna, \& Kear, 1990). El cuestionario consta de 20 ítems, los cuales están representados por un dibujo de la tira cómica Garfield, y se responden en una escala tipo Likert de 4 puntos. Cada ítem está compuesto de una sencilla pregunta o afirmación, seguida de cuatro ilustraciones del gato que muestran diferentes estados emocionales de este, que van desde Muy feliz a Muy Molesto. Las respuestas y sus puntuaciones directas se transforman en rangos percentiles. El rango percentil permite una puntuación total de la actitud general hacia la lectura con dos componentes: la actitud recreativa hacia la lectura y la actitud académica hacia la lectura. El grado de consistencia interna oscila entre 0.74 en la parte de lectura recreativa, en el primer grado, a 0.89 de la escala total en los grados 4to. y 6to. Dicho instrumento fue utilizado en una traducción libre al idioma español realizada por las autoras de esta investigación.

\subsection{Instrumentos cualitativos}

Se utilizaron cuatro técnicas cualitativas: la primera fue la observación no participante correspondiente al Instrumento de Evaluación Psicopedagógica (IEP), que analiza el proceso de enseñanza y aprendizaje en diferentes áreas, como son: los intereses de los 
estudiantes, los estilos y ritmos en el aprendizaje, la práctica de aula, relativa tanto a los estudiantes como a la maestra, y el aspecto socio-afectivo de los estudiantes. En esta oportunidad, a diferencia de los datos cuantitativos, se sistematizó la información emergente y de mayor sentido en cada una de las cuatro escuelas.

La segunda técnica fue la valoración de la percepción y de la actitud de los niños y las niñas hacia la clase de matemática, por medio de la realización de un dibujo libre, técnica utilizada por Borthwick, A. (C. Smith, (Ed.), 2011, en Proceedings of the British Society for Research into Learning Mathematics).

La tercera técnica utilizada fue el grupo de enfoque, dirigido a estudiantes de 1 ro. a 4to. grados de primaria. Los temas del grupo de enfoque se orientaban a dos grandes aspectos: el primero la percepción del niño o niña de su experiencia educativa, y el segundo relativo a su valoración hacia la familia. La cuarta técnica utilizada fue un cuestionario creativo, elaborado por las investigadoras, con el fin de interpelar el sentimiento y la imaginación de las maestras y poder capturar a través de la metáfora su real sentir hacia la labor docente. El instrumento utilizado se denomina la Voz de la Maestra.

\subsection{Procedimiento}

El diseño DISTRAC en la investigación comprendió tres fases de recolección y análisis de los datos, inicial, intermedia y final.

En la primera fase o inicial se recogieron simultáneamente la data cuantitativa, a partir de la aplicación de los instrumentos referidos, y la data cualitativa, por medio a las técnicas expuestas.

Con relación al instrumento "Valoración del Desarrollo Socioafectivo", que constaba de tres secciones, la primera relativa al Ambiente Familiar, la segunda a Valores y Actitudes y la tercera referida a la Afectividad, solo se utilizó esta última en razón de que las dos precedentes implicaban un acceso y conocimiento 
Aurora Andreina Jiménez. Soto, Rocío Hernández. Mella, Patricia Liranzo Soto, Berenice Pacheco-Salazar

más profundo, tanto de la familia como del propio estudiante, aspectos no viables en el lapso de tiempo programado para esta investigación.

Con respecto al cuestionario de actitud hacia la lectura en el nivel básico, denominado ERAS por sus siglas en inglés (The Elementary Reading Attitude Survey), se realizó la modificación de la caricatura de Garfield, por los emoticons o emoticones (representación gráfica de expresión de emociones) utilizada de manera común en las redes sociales y aplicaciones electrónicas como el WhatsApp. Los emoticones utilizados responden a las emociones de Muy Contento/a, Contento/a, Enojada/o y Muy Enojada/o.

La fase intermedia de recolección y análisis de los datos se llevó a cabo en cuatro diferentes momentos, coincidentes con la realización de los Encuentros Psico-afectivos (EPA): Cuerpo Vivencial, Cuerpo que Expresa, Cuerpo que Comunica y Cuerpo que Representa, que comprenden cuatro (4) talleres en total. A través de este proceso se recopilaron e interpretaron datos por medio a distintas técnicas como fueron: observación no participante, observación participativa, y análisis de contenido, a través de diferentes medios como materiales audiovisuales, documentos escritos personales y bitácoras de análisis.

En la fase final, se aplicó nuevamente el instrumento "Valoración del Desarrollo Socio-afectivo".

El Instrumento de Evaluación Psicopedagógica (IEP) no pudo aplicarse nuevamente, por las razones de complejidad de dicho recurso y del ambiente disruptivo y violento encontrado en las aulas. En cuanto al cuestionario de actitud hacia la lectura en el nivel básico (ERAS, por sus siglas en inglés The Elementary Reading Attitude Survey), se tomó la decisión de no aplicarlo al darnos cuenta de que las respuestas emitidas por los niños y niñas entrevistados tendían a dar una impresión favorable de sí mismos/as, en vez de manifestar lo que realmente sentían. Con respecto al dibujo que se refería a la valoración de la percepción y actitud de los niños y niñas hacia la clase de matemática, la 
disposición de no llevarlo a cabo nuevamente obedeció a que más de la mitad de estudiantes no expresó a través del dibujo la instrucción que se le pidió. De ahí que las investigadoras tomaron la determinación de adicionar, a lo estipulado, un tipo de análisis siguiendo aportaciones de la Psicología Profunda, ya que se asume que es posible extraer información significativa de este modo y no repetir el procedimiento.

\section{A modo de conclusión}

Arte y Afectividad en la experiencia escolar inclusiva: un estudio dominicano ha sido una investigación desarrollada con fines exploratorios. Se concentra en descripciones de la experiencia educativa de niños y niñas en interacción con sus docentes, identificando disposiciones personales y expresión de sentimientos, por parte de los primeros, y actitudes, acciones y recursos didácticos, por parte de los segundos. Esto logrado a partir de un enfoque mixto; es decir, desde un análisis que combina lo cuantitativo con lo cualitativo.

De la misma manera, Arte y Afectividad incluye una intervención desarrollada con estrategias lúdicas y artísticas, la cual pretende complementar y apoyar el proceso educativo que se desarrolla en el Nivel Básico de escolaridad.

Tanto la exploración, a través del estudio realizado, así como la estrategia de intervención, han permitido la elaboración de cinco trabajos que aparecerán en otras tantas entregas. Este artículo corresponde a la primera de ellas. En la segunda entrega, se aportan los resultados y el análisis de las herramientas de investigación Instrumento de Evaluación Psicopedagógica (IEP), de Hernández, R., Andújar, C., Scheker, A., Pérez, M. \& Amiama, C. (2000), y el cuestionario creativo Voz de la Maestra, elaborado por las investigadoras, especialmente para este estudio. 
Aurora Andreina Jiménez. Soto, Rocío Hernández. Mella, Patricia Liranzo Soto, Berenice Pacheco-Salazar

\section{Referencias bibliográficas}

Abad, J. (2009). Usos y funciones de las artes en la educación y el desarrollo humano. En Jiménez, L., Aguirre, I., \& Pimentel, L. (Coods) Educación Artística Cultura y Ciudadanía (1-183). Madrid: OEI. Recuperado de http://www.oei.es/metas20 21/EDART2.pdf

Ainscow, M. (2003). Desarrollo de sistemas educativos inclusivo. Reino Unido: The University of Manchester.

Badilla, F. (2011). Arte terapia: Una manera de fortalecer la autoestima. (Tesis de maestría), Santiago de Chile, Universidad de Chile. Recuperado de http://www.tesis.uchile.cl/tesis/uchile/2011 /ar-badilla_f/pdfAmont/ar-badilla_f.pdf

Benítez, I. M. (s. f.). Significado del dibujo infantil. Recuperado de http://www.eduinnova.es/mar09/Significado $\% 20 \mathrm{del} \% 20$ dibujo $\% 20$ infantil.pdf

Booth, T. (s. f.). Haciendo espacio para el desarrollo educacional: valores inclusivos y el indice de inclusión. Recuperado de file://C:/Users / rocio/Downloads/ponencia $\% 20 \mathrm{de} \% 20$ tony $\% 20$ booth $\%$ $20 \% 20$ en $\% 20$ castellano.pdf

Borthwick, A. (2011). Children's perceptions of, and attitudes towards, their mathematics lessons. En C. Smith (Ed.). Proceedings of the British Society for Research into Learning Mathematics 31(1), 37-43. Recuperado de http://www.bsrlm.org.uk/IPs /ip31-1/BSRLM-IP-31-1-07.pdf

Cabezas, C. (2007). Análisis y características del dibujo infantil. Recuperado de https://n-1.cc/file/download/1035141

Escabias, Ma. del M. (2008). Características del dibujo infantil. Revista Enfoques Educativos (pp. 63-73). Recuperado de http://www.enfoqueseducativos.es/enfoques/enfoques_ 23.pdf 
Arte y afectividad en la experiencia escolar inclusiva: Un estudio dominicano

Gardner, H. (1997). La mente no escolarizada. Cómo piensan los niños y cómo deberian enseñar las escuelas. México: Biblioteca del Normalista.

Gardner, H. (1993): Frames of Mind. The Theory of Multiple Intelligences. Gran Bretaña: Fontana Press.

Grajales, A. (2011). Orientación grupal para el bienestar del adulto mayor. (Tesis de maestría), Universidad Autónoma de Yucatán, México. Recuperado de http://posgradofeuady.org.mx/ wpcontent/uploads/2011/09/orientacionBAM.pdf

Hernández, R. (2012). Ambientes afectivos y efectivos de aprendizaje. Recuperado de http://www.uv.mx/personal/rubhernandez/ files/2012/02/Amb_afectivos_efectivos.pdf

Hernández Mella, R. (2014). Aportes de la psicología social al análisis de la condición de sobreedad en la educación. De la exclusión a la oportunidad. (Tesis doctoral, inédita). Madrid: Universidad Complutense de Madrid.

Hernández Mella, R. \& Andujar, C. (1999). Valoración del desarrollo socioafectivo. Instrumento, resultados y conclusiones. Santo Domingo: Secretaría de Estado de Educación.

Hernández Mella, R. \& Andujar, C.; Scheker, A., \& Pérez, M. \& Amiama, C. (2000). Instrumento de evaluación psicopedagógica. Santo Domingo: Secretaría de Estado de Educación.

Hernández Mella, R. \& Pacheco, B. (2012). Vivir feliz como propuesta para un quehacer docente imaginativo e inclusivo. Santo Domingo: OEI y MINERD.

Hernández-Sampieri, R.; Fernández-Collado, C. \& Baptista-Lucio, M. P. (2010). Metodología de la investigación. México: McGrawHill Interamericana Editores.

Jiménez, L. (2010). Arte, cultura y ciudadanía: hacia la construcción de valores. En B. Toro \& A. Tallone (Eds.). Educación, valores 
Aurora Andreina Jiménez. Soto, Rocío Hernández. Mella, Patricia Liranzo Soto, Berenice Pacheco-Salazar

y ciudadanía. Metas educativas 2021 (pp. 203-214). Madrid: OEI \& Fundación SM.

Keat, J. \& Wilburne, J. (2009). The impact of storybooks on kindergarten children's mathematical achievement and approaches to learning. US-China Education Review, 6(7). 61-67. Recuperado de http:/ files.eric.ed.gov/fulltext/ED506319.pdf

McKenna, M. C. \& Kear, D. J. (1990). Measuring attitude toward reading: A new tool for teachers. The Reading Teacher, 43(8), 626-639. Recuperado de http://dx.doi.org/10.1598/RT.43.8.3

Maturana, H. (1990): Emociones y lenguaje en educación y política. Santiago de Chile: Hachette/CED.

Maturana R., H. (1992). El sentido de lo bumano. Santiago de Chile: Hachette y Ediciones Pedagógicas Chilenas.

Mengíbar, C. (2010). El despertar del dibujo. Recuperado de http://www.csicsif.es/andalucia/modules/mod_ense/revista /pdf/Numero_31/CARMEN_MENGIBAR_1.pdf

Morris, Ch. \& Maisto, A. (2005). Introducción a la psicología. México: Pearson Educación. Recuperado de http://books.google. com.do/books?id=5S1 dyNo96zUC\&printsec $=$ frontcover\# $\mathrm{v}=$ onepage\&q\&f$=$ false

Ortego, M. C., López, S. \& Álvarez, M. L. (2011) Tema 4: Las actitudes. Ciencias Psicosociales I. Recuperado de http:/ /ocw. unican.es/ciencias-de-la-salud/ciencias-psicosociales-i/mate riales/bloque-i/tema-4.-las-actitudes/tema-4.-las-actitudes

Piaget, J. (1975, 1986): Seis estudios de psicología. Barcelona: Barral Editores.

Pozzoli, M. T. (2007). Espiritualidad, arte y belleza: Espacios del universo para el desarrollo humano desde el pensamiento complejo. Recuperado de http://www.redalyc.org/articulo.oa?id=30501708 
Arte y afectividad en la experiencia escolar inclusiva: Un estudio dominicano

Prieto, M. A. (2011). Actitudes y valores. Innovación y Experiencias Educativas 41. 1-8. Recuperado de http://www.csicsif.es/ andalucia/modules/mod_ense/revista/pdf/Numero_41/ MIGUEL_ANGEL_PRIETO_BASCON_01.pdf

Restrepo, G. (2009). Contextos afectivos y cognitivos en los procesos de aprendizaje. Revista Complutense de Educación, 20(1) 195-204. Recuperado de http://revistas.ucm.es/index.php/ RCED/article/view/RCED0909120195A/15425

Rodríguez, G. (2010). Sentimientos y actitudes en la escuela. (Capítulo 15, Educación, valores y ciudadanía). Recuperado de http://www. oei.es/metas2021/valoressm.pdf

Sánchez, S. \& Mesa, C. (1998). Actitudes hacia la tolerancia y la cooperación en ambientes multiculturales: evaluación e intervención educativa en un contexto concreto: la ciudad de Melilla. Series Monográficas Eirene Vol. 9. 1-36. Recuperado de http://www.ugr.es/ eirene/publicaciones/item9/eire ne9cap1.pdf

Santiago, E. (2009) Música como estrategia educativa en el proceso enseñanz̧a aprendizaje para el aprovechamiento de los estudiantes del nivel elemental en la Región Educativa de Bayamón. (Tesis de maestría). Río Piedras, Universidad Metropolitana Río Piedras, Puerto Rico. Recuperado de http://www.suagm.edu/umet/biblio teca/UMTESIS/Tesis_Educacion/Adm_Sup_Educativa/ ESantiagoSilva.062309.pdf

Tirado, V. (2008). El proceso de enseñanza aprendizaje a través de las artes plásticas en la educación pre-escolar. (Tesis de grado). Sinaloa, México. Universidad Pedagógica Nacional. Recuperado de http://biblioteca.ajusco.upn.mx/pdf/26368.pdf

Vásquez, J. (2011). El valor del dibujo para la educación infantil. Recuperado de http://www.eduinnova.es/monografias2011 /ene2011/dibujo.pdf 
Aurora Andreina Jiménez. Soto, Rocío Hernández. Mella, Patricia Liranzo Soto, Berenice Pacheco-Salazar

Villamán, M. (2003). Reinventar la Escuela: algunas posibles provocaciones. En Centro Poveda (Eds.). Reinventar la Escuela: ¿Qué Opciones? Reflexiones sobre el futuro de la educación dominicana (p. 67-106). Recuperado de http://www.minerd.gob.do/idec /Docs5/Reinventar $\% 201 \mathrm{a} \% 20$ escuela.pdf

Villanueva, E. (2013). Danza y educación. Propuestas ante los cambios que requiere la actualización curricular. Naturaleza, contenidos y competencias (p. 87-90). [VIII Foro Nacional Educación Artística]. Santo Domingo: Ministerio de Educación de República Dominicana.

Villegas, C. (2010, septiembre). La afectividad como el eje central del encuentro educativo. (Ponencia presentada en Congreso Iberoamericano de Educación Metas Educativas, 2021). Santo Domingo: Organización de los Estados Iberoamericanos para la Educación, la Ciencia y la Cultura. Recuperado de http://www.chubut.edu.ar/descargas/secundaria/congreso/ METAS2021/R0734b_Poster_Villegas.pdf 


\section{Andreína Jiménez}

Es egresada de la carrera de Psicología de Instituto Tecnológico de Santo Domingo (INTEC), maestría en Gerencia Educativa en UNIBE; especialidad en Educación Artística, Cultura y Ciudadanía. Actualmente se desempeña como Técnico Docente Nacional del Área de Educación Artística de la Dirección General de Currículo del Ministerio de Educación; profesora de INTEC. Bailarina, coreógrafa y profesora de Danza Contemporánea con estudios en México y Estados Unidos, obtuvo cinco nominaciones a Premios Casandra y reconocimiento a su trayectoria por el Ministerio de Cultura.

Correo electrónico: aajsoto@gmail.com

\section{Rocío Hernández Mella}

Profesora-investigadora del Área de Ciencias Sociales y Humanidades del Instituto Tecnológico de Santo Domingo, INTEC. Doctora en Psicología Social; maestría en Planificación y Administración de la Educación; especialización en Psicología Educativa y licenciatura en Psicología. Consultora en Psicología y Educación para OEI-República Dominicana y el MINERD; coordinadora Educativa del Centro Jung Santo Domingo y vicepresidenta de la Fundación Tonucci: Educando para la Diversidad. Entre sus publicaciones recientes están: Las diosas sometidas: Autoconcepto en mujeres de grupos vulnerables. Encuentros afectivo-participativos y sus efectos en el self (junto a Patricia Liranzo, 2014), 
Aurora Andreina Jiménez. Soto, Rocío Hernández. Mella, Patricia Liranzo Soto, Berenice Pacheco-Salazar

Vivir feliz como propuesta para un quehacer docente imaginativo e inclusivo (junto a Berenice Pacheco, 2012); El arte salva (capítulo, 2012). Es coautora de los artículos ya publicados: "La sobreedad escolar: de la exclusión a la oportunidad" (2011); "Nuevas miradas psicológicas al 'ser mujer': despertar, transgredir y renacer en el arte” (2009); "De la culpa a la redención: hacia una nueva psicología” (2009).

Correo electrónico: rocio.hernandez@intec.edu.do

\section{Patricia Liranzo}

Es psicóloga clínica, profesora e investigadora del Área de Ciencias Sociales y Humanidades del Instituto Tecnológico de Santo Domingo (INTEC). Posee una maestría en el área de Psicología Clínica por la Universidad Autónoma de Santo Domingo; licenciatura en Psicología; curso Experto en Peritaje Psicológico e Intervención Judicial; curso en Diagnóstico y Rehabilitación Neuropsicológica en Neurología Conductual; curso en Evaluación e Intervención Psicofisiológica en Medicina Conductual.

Además se ha desempeñado como facilitadora en OEI-República Dominicana; coordinadora de diplomados y asistente de investigación en el INTEC. Entre sus publicaciones están: Las diosas sometidas: autoconcepto en mujeres de grupos vulnerables. Encuentros afectivo-participativos y sus efectos en el self (2013); Apego y autoestima estudiantes femeninas en Psicologia (2012); El proceso de descentralización escolar y sus condiciones de posibilidad (2006).

Correo electrónico: patricia.liranzo@intec.edu.do 


\section{Berenice Pacheco-Salazar}

Es doctoranda en Educación por la Universidad de Sevilla. Es licenciada en psicología con maestría en Género, Investigación y Desarrollo. Especialista para la Oficina Dominicana de la Organización de Estados Iberoamericanos para la Educación, la Ciencia y la Cultura (OEI) donde coordina las líneas de Cultura Escrita, Educación en Valores y para la Ciudadanía, y Educación Artística y Cultura. Docente de la Universidad INTEC. Es autora y coautora de diversas publicaciones sobre las temáticas de promoción de la lectura, derechos humanos, educación en valores e inclusión y diversidad. Es poeta, con dos libros publicados y reconocimientos en Argentina y Perú. Actualmente trabaja en su proyecto de tesis doctoral sobre la temática de la violencia escolar en el nivel primario en República Dominicana.

Correo electrónico: bereniceps@gmail.com

Recibido: 25-05-2015

Aprobado: 09-12-2015 
